\title{
ANTIOXIDATIVE LOW MOLECULAR WEIGHT EXTRACTIVES FROM TRIPLOID POPULUS TOMENTOSA XYLEM
}

\author{
Chuan-Ling Si, ${ }^{\text {ab,c, },{ }^{*}}$ Yi-Yuan Lu, ${ }^{\mathrm{a}}$ Yu Zhang, ${ }^{\mathrm{a}} \mathrm{Jie} \mathrm{Xu},{ }^{\mathrm{a}}$ Pan-Pan Qin, ${ }^{\mathrm{a}}$ Run-Cang Sun,
} and Yong-Hao $\mathrm{Ni}^{\mathrm{c}}$

Triploid Populus tomentosa Carr. (Salicaceae) is a good alternative to meet the increasing need of the global pulp and paper industry. Meanwhile, the xylem of this species could be a useful bioresource to develop low molecular extractives with significant bioactive potential. In the present work, a phytochemical investigation on aqueous $\mathrm{EtOH}$ extractives of Triploid $P$. tomentosa xylem, by systematical performance of Sephadex LH-20 open column chromatography and Thin Layer Chromatography (TLC), resulted in the isolation of two phenolic acids ( $\rho$ coumaric acid (I) and caffeic acid (II)), two flavonoids (apigenin (III) and luteolin (IV)), and three phenolic glucosides (salicortin (V), salireposide (VI) and populoside (VII)). The structure elucidation and determination of the isolated extractives were based on their spectroscopical data and physiochemical evidences. This was the first time to report the low molecular weight extractives of Triploid $P$. tomentosa. Various low molecular weight extractives from Triploid $P$. tomentosa xylem exhibited significant antioxidative activities by DPPH and hydroxyl radical scavenging assays.

Keywords: Triploid Populus tomentosa Carr.; Xylem; Low molecular weight extractives; Column chromatography; Spectroscopical data; Antioxidative activity

Contact information: a: Tianjin Key Laboratory of Pulp and Paper, College of Material Science and Chemical Engineering, Tianjin University of Science and Technology, Tianjin 300457, China; b: Key Laboratory of Cellulose and Lignocellulosics Chemistry, Guangzhou Institute of Chemistry, Chinese Academy of Sciences, Guangzhou 510650, China; c: Limerick Pulp and Paper Centre, University of New Brunswick, Fredericton E3B 5A3, Canada; d: Institute of Biomass Chemistry and Technology, Beijing Forestry University, Beijing 100083, China. *Corresponding author: sichli@tust.edu.cn (C.L. Si)

\section{INTRODUCTION}

Chinese forest researchers have been making significant efforts to develop fastgrowing trees due to the extreme shortage of xylem resource in the pulp and paper making industry. Triploid Populus tomentosa Carr., the cloned hardware poplar species from Populus tomentosa Carr., has been receiving the most attention (Zhang et al. 2010; Yang et al. 2006). Its chemical profile from a pulping point of view was reported (Yang et al. 2006). Zhang et al. (2010) have fractionally isolated and characterized the lignin and hemicelluloses of this cloned species previously. However, chemical constituents of low molecular weight extractives and biological activities of Triploid P. tomentosa have never been reported to date, though poplars have been widely used in folk medicines for the treatment of various diseases, such as diarrhea, pox, paralysis, variola, and pulmonary disorders (Si et al. 2010, 2009a, 2009b, 2009c; Zhang et al. 2006). 
Reactive oxygen species, including superoxide anion, hydroxyl radical, and hydrogen peroxide, are chemically reactive free radicals derived from oxygen, which have been implicated in various of diseases due to the oxidative damage to DNA, lipids, and proteins, and which can result in failure of cellular functions (Brawek et al. 2010; Lane 2008). Fortunately, medical and cosmetic use or dietary consumption of antioxidative compounds will reduce oxidative damage (Hosoya et al. 2008). Some synthetic antioxidants show side effects; for example, butylated hydroxyanisole (BHA) has been reported to increase incidences of neoplasias (Tehila et al. 2007). Therefore, considerable effort has been directed towards identifying safe and natural antioxidants that can protect against oxidative stress. Natural compounds, such as phenolic acids, flavonoids, and phenolic glucosides, commonly found in many plants, have shown a wide range of biological and pharmacological activities, including antioxidative activity chelation of metals, anti-HIV, and antiplasmodial activities (Casano et al. 2010; Jung et al. 2010; Neo et al. 2010).

In the present study, a phytochemical study of $95 \% \mathrm{EtOH}$ extractives of triploid $P$. tomentosa xylem, by repeated Sephadex LH-20 open column chromatography and Thin Layer Chromatography (TLC), led to the isolation of two phenolic acids ( $\rho$ coumaric acid (I) and caffeic acid (II)), two flavonoids (apigenin (III) and luteolin (IV)), and three phenolic glucosides (salicortin (V), salireposide (VI), and populoside (VII)). The structures of the isolated phenolic compounds were elucidated and determined on the basis of their physiochemical and spectroscopical evidences $\left({ }^{1} \mathrm{H}-\right.$ and ${ }^{13} \mathrm{C}-\mathrm{NMR}$, as well as MS). The antioxidative activities of the isolated extractives of Triploid $P$. tomentosa xylem were assessed by DPPH and hydroxyl radical scavenging assays.

\section{EXPERIMENTAL}

\section{Plant Materials}

Xylem of Triploid P. tomentosa was obtained from Professor Qun Li's research group of Tianjin Key Laboratory of Pulp \& Paper, College of Material Science and Chemical Engineering, Tianjin University of Science and Technology, China, in June of 2009, and was authenticated by Professor Zhong Liu, Tianjin Key Laboratory of Pulp \& Paper. A voucher specimen (No. 2009-10) has been deposited at the herbarium of Tianjin Key Laboratory of Pulp \& Paper.

\section{Equipment and Reagents}

Column chromatography was carried out with Sephadex LH-20 (Merck). Eluents were collected with a fraction collector (SBS-160). TLC analyses were conducted on DCPlastikfolien Cellulose F (Merck) plates and developed with $t$-BuOH-HOAc- $\mathrm{H}_{2} \mathrm{O}(3: 1: 1$, $\mathrm{v} / \mathrm{v}$, solvent A) and $\mathrm{HOAc}-\mathrm{H}_{2} \mathrm{O}(3: 47, \mathrm{v} / \mathrm{v}$, solvent B). Visualization and detection were performed by UV light (254 and $365 \mathrm{~nm}$ ) and then by spraying with $1 \%$ ethanolic $\mathrm{FeCl}_{3}$ solution followed by heating.

${ }^{1} \mathrm{H}-\mathrm{NMR},{ }^{13} \mathrm{C}-\mathrm{NMR}$, DEPT, and correlation NMR spectra such as $\mathrm{HMBC}$ and $\mathrm{HMQC}$ were recorded in $\mathrm{CD}_{3} \mathrm{OD},\left(\mathrm{CD}_{3}\right)_{2} \mathrm{SO}$, or $\left(\mathrm{CD}_{3}\right)_{2} \mathrm{CO}$ with TMS as an internal standard using a Bruker Avance DPX 400 spectrometer at the operating frequency of 400 
$\mathrm{MHz}\left({ }^{1} \mathrm{H}\right)$ and $100 \mathrm{MHz}\left({ }^{13} \mathrm{C}\right)$ at State Key Laboratory of Pulp and Paper Engineering, South China University of Technology, China. Positive FAB MS and EI MS spectroscopy were done with a Micromass Autospec M363 spectrometer at Limerick Pulp and Paper Center, University of New Brunswick, Canada.

\section{Extraction and Fractionation}

Air-dried and finely powered Triploid P. tomentosa xylem $(3.2 \mathrm{~kg})$ was extracted five times in $95 \%$ aqueous EtOH solution for more than one week at room temperature. After combination, filtration, concentration, and removing EtOH with a rotary evaporator in vacuo, the residue mixture was successively fractionated and freeze-dried to give fractions soluble in $n$-hexane $(6.3 \mathrm{~g}$, yield $0.20 \%), \mathrm{CH}_{2} \mathrm{Cl}_{2}(7.5 \mathrm{~g}$, yield $0.23 \%)$, EtOAc (25.1 g, yield 0.78\%), $\mathrm{BuOH}\left(19.6 \mathrm{~g}\right.$, yield $0.61 \%$ ), and $\mathrm{H}_{2} \mathrm{O}(131.2 \mathrm{~g}$, yield $4.1 \%$ ). Soluble fractions were kept in dark, cool, and sealed vials.

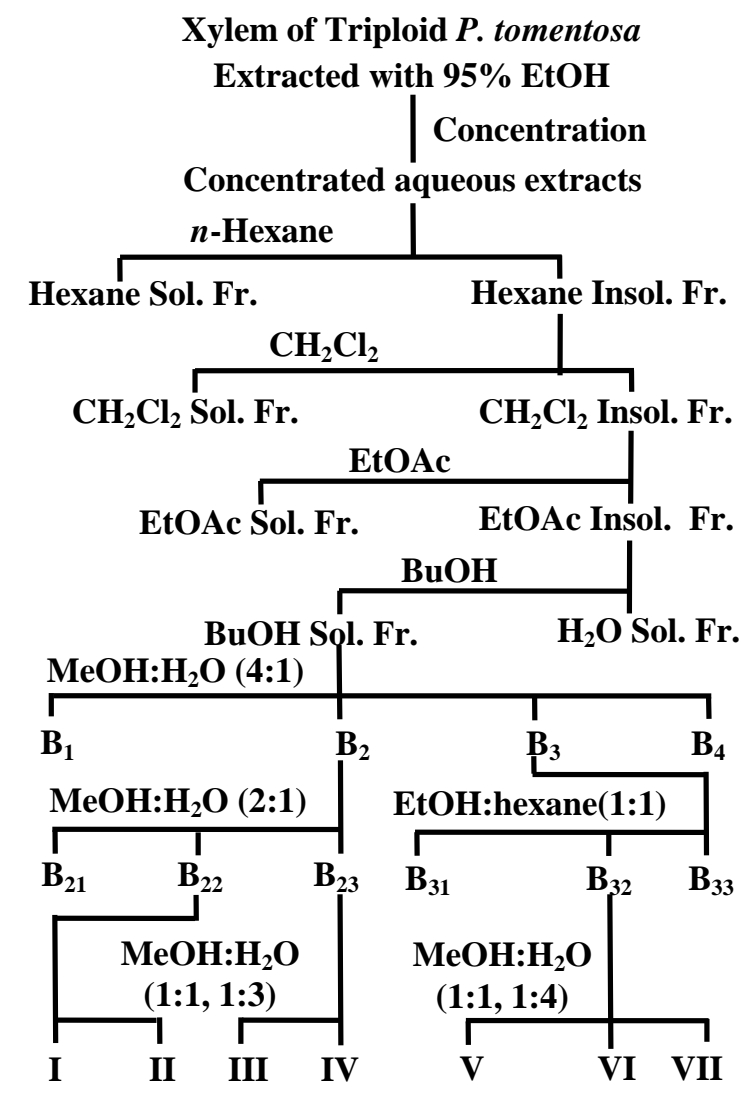

Fig. 1. Extraction, fractionation and isolation procedures of low molecular weight extractives from xylem of Triploid $P$. tomentosa

\section{Isolation of Extractives}

As shown in Fig. 1, a portion of the above freeze-dried $\mathrm{BuOH}$ soluble fraction powder (19.2 g) was subjected to a Sephadex LH-20 column with $\mathrm{MeOH}-\mathrm{H}_{2} \mathrm{O}(4: 1, \mathrm{v} / \mathrm{v})$ used as elutent to give four fractions labelled as $\mathrm{B}_{1}(1.3 \mathrm{~g}), \mathrm{B}_{2}(7.6 \mathrm{~g}), \mathrm{B}_{3}(9.1 \mathrm{~g})$, and $\mathrm{B}_{4}$ $(1.1 \mathrm{~g})$, which were guided and monitored by TLC performance. Fraction $\mathrm{B}_{2}$ was 
resubjected to Sephadex LH-20 column chromatography using $\mathrm{MeOH}-\mathrm{H}_{2} \mathrm{O}(2: 1, \mathrm{v} / \mathrm{v})$ for further purification to yield three subfractions, namely $\mathrm{B}_{21}, \mathrm{~B}_{21}$, and $\mathrm{B}_{23}$. The second $\left(\mathrm{B}_{22}\right.$, $3.8 \mathrm{~g})$ and third subfractions $\left(\mathrm{B}_{23}, 2.9 \mathrm{~g}\right)$ were eluted with $\mathrm{MeOH}-\mathrm{H}_{2} \mathrm{O}(1: 1$ and 1:3, v/v) likewisely, and compounds I (61 mg), II (43 mg), III (112 mg), and IV (88 mg) were obtained. Fraction $\mathrm{B}_{3}$ was also applied to a Sephadex LH-20 column with EtOH-hexane $(1: 1, \mathrm{v} / \mathrm{v})$ as eluting mixtures for further purification to give three subfractions that will be referred to as $\mathrm{B}_{31}(1.2 \mathrm{mg}), \mathrm{B}_{32}(7.2 \mathrm{~g})$ and $\mathrm{B}_{33}(0.6 \mathrm{~g})$. When treated with $\mathrm{MeOH}-\mathrm{H}_{2} \mathrm{O}$ (1:1 and $1: 4, \mathrm{v} / \mathrm{v})$, compounds V (48 $\mathrm{mg})$, VI (72 mg) and VII (36 mg) were obtained from $\mathrm{B}_{32}$ as yellowish powders.

\section{Antioxidative Activity Assays}

$D P P H$ radical scavenging assay

DPPH radical scavenging assay was carried out according to the procedure described by Blois (1958) with minor suitable modification. $\mathrm{MeOH}$ solutions $(4 \mathrm{ml})$ of samples at different concentrations were added to a solution of DPPH $\left(1.5 \times 10^{-4} \mathrm{M}, 1 \mathrm{ml}\right)$ in $\mathrm{MeOH}$. After mixing gently and standing at room temperature for $30 \mathrm{~min}$, the optical density was measured at $517 \mathrm{~nm}$ with a UV-visible spectrophotometer (UV-1600, Rayleigh). The results were calculated by taking the mean of all triplicated values. $\mathrm{IC}_{50}$ values were obtained through extrapolation from concentration of sample necessary to scavenge $50 \%$ of the DPPH free radicals. $\alpha$-Tochopherol, an excellent natural antioxidant, was used as a positive control.

\section{Hydroxyl radical scavenging assay}

A hydroxyl radical scavenging assay was performed using a standard method (Chung et al. 1997). The Fenton reaction mixture consisted of $200 \mu \mathrm{L}$ of $\mathrm{FeSO}_{4} \cdot 7 \mathrm{H}_{2} \mathrm{O}$ $(10 \mathrm{mM}), 2$-deoxyribose $(10 \mathrm{mM})$ and EDTA $(10 \mathrm{mM}) .200 \mu \mathrm{L}$ of sample and $1 \mathrm{~mL}$ of $0.1 \mathrm{M}$ phosphate buffer ( $\mathrm{pH} 7.4$ ) were added to make up a total volume of $1.8 \mathrm{ml}$. Then, $200 \mathrm{ul}$ of $10 \mathrm{mM} \mathrm{H}_{2} \mathrm{O}_{2}$ was added and the reaction mixture was cultured at $37{ }^{\circ} \mathrm{C}$ for $4 \mathrm{~h}$. After the incubation, $1 \mathrm{~mL}$ of $1 \%$ TBA and $1 \mathrm{~mL}$ of $2.8 \%$ TCA were added and the solution was placed in a boiling water bath for $10 \mathrm{~min}$. After cooling, the solution was centrifuged for $5 \mathrm{~min}(3000 \mathrm{rpm})$ and the absorbance was measured at $532 \mathrm{~nm}$ with a UV-visible spectrophotometer (UV-1600, Rayleigh). Each assay was carried out in triplicate. $\alpha$-Tochopherol was employed as a positive control. The hydroxyl radical scavenging activity of the sample was calculated using following formula,

$$
\text { Scavenging activity }(\%)=\left[1-\frac{A_{1}-A_{2}}{A_{0}}\right] \times 100 \%
$$

where $A_{0}$ is the absorbance of the control (blank, without extract), $A_{1}$ is the absorbance in the presence of the extract, and $A_{2}$ is the absorbance without 2-deoxyribose).

\section{Statistical Analyses}

All results were presented as mean $\pm \mathrm{SD}$, and the statistical analyses were subjected to one-way analysis of variation (ANOVA). The significance of difference between means was determined by Tukey's test $(P<0.05)$ for multiple comparisons. 


\section{RESULTS AND DISCUSSION}

\section{Low Molecular Weight Extractives Isolation and Identification}

Based on a successive Sephadex LH-20 column chromatographic separation of a portion of $\mathrm{BuOH}$ soluble fraction that was extracted with $95 \% \mathrm{EtOH}$ from xylem of Triploid P. tomentosa aided by TLC, seven yellowish low molecular weight natural extractives, as shown in Fig. 2, including two phenolic acids, $\rho$-coumaric acid (I), and caffeic acid (II) (Kim et al. 2002; Kwon et al. 1999), two flavonoids, apigenin (III) and luteolin (IV) (Agrawal 1989; Markham and Chari 1982), and three phenolic glucosides, salicortin (V), salireposide (VI), and populoside (VII) (Si et al. 2010, 2009a, 2009b, 2009c), were isolated, and their structure elucidation was established on the basis of their physiochemical and spectroscopic data. To the best of our knowledge, this was the first time of report natural compounds with low molecular weight from Triploid P. tomentosa xylem.

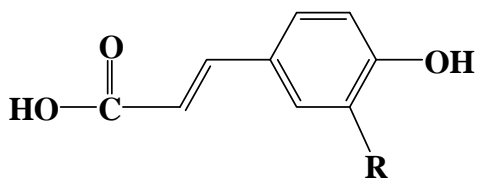

$\mathrm{R}=\mathrm{H}: \rho$-Coumaric acid (I)

$\mathrm{R}=\mathrm{H}$ : Caffeic acid (II)<smiles>[R]c1cc(-c2cc(=O)c3c(O)cc(O)cc3o2)ccc1O</smiles>

$\mathrm{R}=\mathrm{H}$ : Apigenin (III)

$\mathrm{R}=\mathrm{OH}$ : Luteolin (IV)

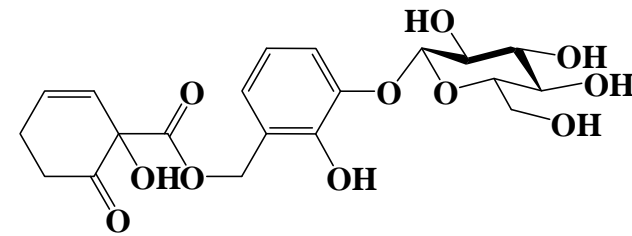

Salicortin (V)<smiles>O=C(OCc1cc(O)ccc1OC(O)C(O)C(O)CO)c1ccccc1</smiles>

Salireposide (VI)<smiles>O=C(/C=C/c1ccc(O)c(O)c1)OCc1ccccc1O[C@@H](O)[C@@H](O)[C@H](O)[C@H](O)CO</smiles>

Populoside (VII)

Fig. 2. Structures of the isolated low molecular weight extractives (I-VII) from Triploid $P$. tomentosa xylem 


\section{Compound I ( $\rho$-Coumaric acid)}

Yellowish amorphous powder; $\mathrm{FeCl}_{3}$ test: Positive (dark brown); $\mathrm{R}_{f}$ : 0.86 (solvent A) and 0.40 (solvent B); EI-MS: Calculated for $\mathrm{C}_{9} \mathrm{H}_{8} \mathrm{O}_{3}$ 164; Found [M] ${ }^{+} \mathrm{m} / z$ 164, [M$\mathrm{OH}]^{+} m / z 147$ and $[\mathrm{M}-\mathrm{COOH}]^{+} m / z$ 119. ${ }^{1} \mathrm{H}-\mathrm{NMR}\left(400 \mathrm{MHz}, \delta, \mathrm{CD}_{3} \mathrm{OD}\right): 6.20(1 \mathrm{H}, \mathrm{d}$, $\mathrm{J}=15.9 \mathrm{~Hz}, \mathrm{H}-8), 6.80(2 \mathrm{H}, \mathrm{d}, \mathrm{J}=8.2 \mathrm{~Hz}, \mathrm{H}-3,5), 7.44(2 \mathrm{H}, \mathrm{d}, \mathrm{J}=8.2 \mathrm{~Hz}, \mathrm{H}-2,6), 7.60(1 \mathrm{H}$, $\mathrm{d}, \mathrm{J}=15.9 \mathrm{~Hz}, \mathrm{H}-7) .{ }^{13} \mathrm{C}-\mathrm{NMR}\left(100 \mathrm{MHz}, \delta, \mathrm{CD}_{3} \mathrm{OD}\right): 115.54$ (C-8), 116.77 (C-3,5), 127.21 (C-1), 131.06 (C-2,6), 146.64 (C-7), 161.10 (C-4), 171.02 (C-9).

\section{Compound II (Caffeic acid)}

Yellowish amorphous powder; $\mathrm{FeCl}_{3}$ test: Positive (dark brown); $\mathrm{R}_{f}: 0.76$ (solvent A) and 0.33 (solvent B); EI-MS: Calculated for $\mathrm{C}_{9} \mathrm{H}_{8} \mathrm{O}_{4}$ 180; Found [M] ${ }^{+} \mathrm{m} / z$ 180, [M$\mathrm{OH}]^{+} m / z 163$ and $[\mathrm{M}-\mathrm{COOH}]^{+} m / z$ 135. ${ }^{1} \mathrm{H}-\mathrm{NMR}\left(400 \mathrm{MHz}, \delta, \mathrm{CD}_{3} \mathrm{OD}\right): 6.20(1 \mathrm{H}, \mathrm{d}$, $\mathrm{J}=16.1 \mathrm{~Hz}, \mathrm{H}-8), 6.77(1 \mathrm{H}, \mathrm{d}, \mathrm{J}=8.0 \mathrm{~Hz}, \mathrm{H}-5), 6.93(1 \mathrm{H}, \mathrm{dd}, \mathrm{J}=2.0 \mathrm{~Hz}$ and $\mathrm{J}=8.0 \mathrm{~Hz}, \mathrm{H}-6)$, $7.04(1 \mathrm{H}, \mathrm{d}, \mathrm{J}=2.0 \mathrm{~Hz}, \mathrm{H}-2), 7.53(1 \mathrm{H}, \mathrm{d}, \mathrm{J}=16.1 \mathrm{~Hz}, \mathrm{H}-7) .{ }^{13} \mathrm{C}-\mathrm{NMR}(100 \mathrm{MHz}, \delta$, $\mathrm{CD}_{3} \mathrm{OD}$ ): 115.11 (C-8), 115.65 (C-5), 116.51 (C-2), 122.88 (C-6), 127.84 (C-1), 146.81 (C-3), 147.00 (C-7), 149.46 (C-4), 171.16 (C-9).

\section{Compound III (Apigenin)}

Yellowish amorphous powder; $\mathrm{FeCl}_{3}$ test: Positive (dark brown); $\mathrm{R}_{f}: 0.77$ (solvent A) and 0.05 (B); EI-MS: Calculated for $\mathrm{C}_{15} \mathrm{H}_{10} \mathrm{O}_{5} 270$; Found $[\mathrm{M}]^{+} \mathrm{m} / z 270,\left[\mathrm{~A}_{1}+\mathrm{H}\right]^{+} \mathrm{m} / z$ 153, $\left[\mathrm{A}_{1}\right]^{+} \mathrm{m} / z 152$ and $\left[\mathrm{B}_{1}\right]^{+} \mathrm{m} / z$ 118. ${ }^{1} \mathrm{H}-\mathrm{NMR}\left(400 \mathrm{MHz}, \delta,\left(\mathrm{CD}_{3}\right)_{2} \mathrm{SO}\right): 6.20(1 \mathrm{H}, \mathrm{d}, \mathrm{J}=1.7$ Hz, H-6), 6.49 (1H, d, J=1.7 Hz, H-8), 6.79 (1H, s, H-3), 6.93 (2H, d, J=8.7 Hz, H-3',5'), $7.93\left(2 \mathrm{H}, \mathrm{d}, \mathrm{J}=8.7 \mathrm{~Hz}, \mathrm{H}-2^{\prime}, 6^{\prime}\right) .{ }^{13} \mathrm{C}-\mathrm{NMR}\left(100 \mathrm{MHz}, \delta,\left(\mathrm{CD}_{3}\right)_{2} \mathrm{SO}\right): 94.82(\mathrm{C}-8), 99.69$ (C-6), 103.67 (C-3), 104.52 (C-10), 116.81 (C-3',5'), 122.01 (C-1'), 129.33 (C-2',6'), 158.16 (C-9), 162.03 (C-5), 162.30 (C-4'), 164.59 (C-2), 165.03 (C-7), 181.60 (C-4).

\section{Compound IV (Luteolin)}

Yellowish amorphous powder; $\mathrm{FeCl}_{3}$ test: Positive (dark brown); $\mathrm{R}_{f}$ : 0.58 (solvent A) and 0.08 (solvent B); EI-MS: Calculated for $\mathrm{C}_{15} \mathrm{H}_{10} \mathrm{O}_{6} 286$; Found $[\mathrm{M}]^{+} m / z$ 286, [A $\left.\mathrm{A}_{1}\right]^{+}$ $m / z$ 152, $\left[\mathrm{A}_{1}+\mathrm{H}\right]^{+} m / z 153$ and $\left[\mathrm{B}_{1}\right]^{+} m / z 134 .{ }^{1} \mathrm{H}-\mathrm{NMR}\left(400 \mathrm{MHz}, \delta,\left(\mathrm{CD}_{3}\right)_{2} \mathrm{CO}\right): 6.26(1 \mathrm{H}$, d, J=1.9 Hz, H-6), $6.53(1 \mathrm{H}, \mathrm{d}, \mathrm{J}=1.9 \mathrm{~Hz}, \mathrm{H}-8), 6.59(1 \mathrm{H}, \mathrm{s}, \mathrm{H}-3), 7.02(1 \mathrm{H}, \mathrm{d}, \mathrm{J}=8.4 \mathrm{~Hz}$, $\left.\mathrm{H}^{-5}\right), 7.48\left(1 \mathrm{H}, \mathrm{dd}, \mathrm{J}=2.0 \mathrm{~Hz}\right.$ and $\left.\mathrm{J}=8.4 \mathrm{~Hz}, \mathrm{H}-6^{\prime}\right), 7.52\left(1 \mathrm{H}, \mathrm{d}, \mathrm{J}=2.0 \mathrm{~Hz}, \mathrm{H}-2^{\prime}\right) .{ }^{13} \mathrm{C}-$ NMR (100 MHz, $\left.\delta,\left(\mathrm{CD}_{3}\right)_{2} \mathrm{CO}\right)$ : 94.72 (C-8), 99.72 (C-6), 104.21 (C-3), 105.35 (C-10), 114.14 (C-2'), 116.64 (C-5'), 120.16 (C-6'), 123.77 (C-1'), 146.49 (C-3'), 150.11 (C-4'), 158.79 (C-9), 163.38 (C-5), 164.92 (C-2), 165.16 (C-7), 183.07 (C-4).

\section{Compound V (Salicortin)}

Yellowish amorphous powder; $\mathrm{FeCl}_{3}$ test: Positive (dark brown); $\mathrm{R}_{f}$ : 0.69 (solvent A) and 0.45 (solvent B); Positive FAB-MS: Calculated for $\mathrm{C}_{20} \mathrm{H}_{24} \mathrm{O}_{10}$ 424; Found $[\mathrm{M}+\mathrm{Na}]^{+} \mathrm{m} / z 447$ and $[\mathrm{M}+\mathrm{H}]^{+} m / z$ 425. ${ }^{1} \mathrm{H}-\mathrm{NMR}$ NMR (400 MHz, $\left.\delta, \mathrm{CD}_{3} \mathrm{OD}\right): 2.45-$ 2.25 (2H, m, Ha-4", Ha-5"), 2.61-2.70 (1H, m, Hb-4"), 2.84-2.92 (1H, m, Hb-5"), 3.35$3.50\left(4 \mathrm{H}, \mathrm{m}, \mathrm{H}-2^{\prime}, 3^{\prime}, 4^{\prime}, 5^{\prime}\right), 3.69\left(1 \mathrm{H}, \mathrm{dd}, \mathrm{J}=5.0,5.2\right.$ and $\left.12.0 \mathrm{~Hz}, \mathrm{Ha}-66^{\prime}\right), 3.88(1 \mathrm{H}, \mathrm{dd}, \mathrm{J}$ $=1.8,1.9$ and $\left.12.1 \mathrm{~Hz}, \mathrm{H}_{\mathrm{b}}-6^{\prime}\right), 4.92\left(1 \mathrm{H}, \mathrm{d}, \mathrm{J}=7.6\right.$ and $\left.2.8 \mathrm{~Hz}, \mathrm{H}-1^{\prime}\right), 5.26(1 \mathrm{H}, \mathrm{d}, \mathrm{J}=12.4$ $\mathrm{Hz}, \mathrm{Ha}-7), 5.37$ (1H, d, J=12.4 Hz, H $\left.\mathrm{b}^{-7}\right), 5.75$ (1H, dt, J =1.6, 1.8, 1.7 and $\left.9.7 \mathrm{~Hz}, \mathrm{H}-2 "\right)$, $6.14(1 \mathrm{H}, \mathrm{dt}, \mathrm{J}=4.1,3.4,3.9$ and $9.8 \mathrm{~Hz}, \mathrm{H}-3 "), 7.01(1 \mathrm{H}, \mathrm{t}, \mathrm{J}=7.4$ and $7.4 \mathrm{~Hz}, \mathrm{H}-4), 7.20$ 
$(1 \mathrm{H}, \mathrm{d}, \mathrm{J}=8.1 \mathrm{~Hz}, \mathrm{H}-6), 7.31(2 \mathrm{H}, \mathrm{m}, \mathrm{H}-3,5) .{ }^{13} \mathrm{C}-\mathrm{NMR}\left(100 \mathrm{MHz}, \delta, \mathrm{CD}_{3} \mathrm{OD}\right): 27.28$ (C4"), 36.91 (C-5"), 62.56 (C-6'), 64.44 (C-7), 71.37 (C-4'), 74.98 (C-2'), 78.06 (C-3'), 78.24 (C-5'), 79.24 (C-1"), 102.52 (C-1'), 116.67 (C-6), 123.46 (C-4), 126.16 (C-2), 129.38 (C-3"), 130.69 (C-5), 131.10 (C-3), 133.38 (C-2"), 157.01 (C-1), 171.58 (C-7"), 207.45 (C-6").

\section{Compound VI (Salireposide)}

Yellowish amorphous powder; $\mathrm{FeCl}_{3}$ test: Positive (dark brown); $\mathrm{R}_{f}$ : 0.86 (solvent A) and 0.67 (B); Positive FAB-MS: Calculated for $\mathrm{C}_{20} \mathrm{H}_{22} \mathrm{O}_{9} 406$; Found $[\mathrm{M}+\mathrm{Na}]^{+} \mathrm{m} / \mathrm{z}$ 429 and $[\mathrm{M}+\mathrm{H}]^{+} \mathrm{m} / z$ 407. ${ }^{1} \mathrm{H}-\mathrm{NMR}$ (400 MHz, $\left.\delta, \mathrm{CD}_{3} \mathrm{OD}\right): 3.37$ (2H, m, H-4', 5'), 3.44 $\left(2 \mathrm{H}, \mathrm{m}, \mathrm{H}-2^{\prime}, 3^{\prime}\right), 3.68\left(1 \mathrm{H}, \mathrm{dd}, \mathrm{J}=5.0\right.$ and $\left.12.0 \mathrm{~Hz}, \mathrm{H}_{\mathrm{a}}-6^{\prime}\right), 3.87\left(1 \mathrm{H}, \mathrm{d}, \mathrm{J}=11.4 \mathrm{~Hz}, \mathrm{H}_{\mathrm{b}}-6^{\prime}\right)$, $4.78\left(1 \mathrm{H}, \mathrm{d}, \mathrm{J}=7.5 \mathrm{~Hz}, \mathrm{H}-1^{\prime}\right), 5.42\left(1 \mathrm{H}, \mathrm{d}, \mathrm{J}=13.0 \mathrm{~Hz}, \mathrm{H}_{\mathrm{a}}-7\right), 5.51\left(1 \mathrm{H}, \mathrm{d}, \mathrm{J}=13.0 \mathrm{~Hz}, \mathrm{H}_{\mathrm{b}^{-}}\right.$ 7), $6.72(1 \mathrm{H}, \mathrm{dd}, \mathrm{J}=3.0$ and $8.7 \mathrm{~Hz}, \mathrm{H}-5), 6.86(1 \mathrm{H}, \mathrm{d}, \mathrm{J}=3.0 \mathrm{~Hz}, \mathrm{H}-3), 7.12(1 \mathrm{H}, \mathrm{d}, \mathrm{J}=8.9$ $\mathrm{Hz}, \mathrm{H}-6), 7.48$ (2H, t, J=7.8, 7.6 and $7.7 \mathrm{~Hz}, \mathrm{H}-3 ", 5 "), 7.61(1 \mathrm{H}, \mathrm{t}, \mathrm{J}=7.4$ and $7.4 \mathrm{~Hz}, \mathrm{H}-$ 4"), $8.06\left(2 \mathrm{H}, \mathrm{dd}, \mathrm{J}=1.4\right.$ and $\left.7.8 \mathrm{~Hz}, \mathrm{H}-2^{\prime \prime}, 6 "\right) .{ }^{13} \mathrm{C}-\mathrm{NMR}\left(100 \mathrm{MHz}, \delta, \mathrm{CD}_{3} \mathrm{OD}\right): 62.62$ (C-6'), 63.24 (C-7), 71.42 (C-4'), 75.06 (C-2'), 78.09 (C-3'), 78.18 (C-5'), 104.39 (C-1'), 116.25 (C-3), 116.62 (C-5), 119.32 (C- 6), 128.76 (C- 1"), 129.66 (C-3",5"), 130.68 (C2",6"), 131.52 (C-2), 134.37(C-4"), 149.99 (C-4), 154.06 (C-1), 168.09 (C-7").

\section{Compound VII (Populoside)}

Yellowish amorphous powder; $\mathrm{FeCl}_{3}$ test: Positive (dark brown); $\mathrm{R}_{f}$ : 0.80 (solvent A) and 0.44 (solvent B); Positive FAB-MS: Calculated for $\mathrm{C}_{22} \mathrm{H}_{24} \mathrm{O}_{10}$ 448; Found $[\mathrm{M}+\mathrm{Na}]^{+} m / z 471$ and $[\mathrm{M}+\mathrm{H}]^{+} m / z$ 449. ${ }^{1} \mathrm{H}-\mathrm{NMR}\left(400 \mathrm{MHz}, \delta, \mathrm{CD}_{3} \mathrm{OD}\right): 3.3-3.5(4 \mathrm{H}$, br m, H-2', 3', 4', 5'), $3.70(1 \mathrm{H}$, dd, J=5.1 and $12.0 \mathrm{~Hz}$, Ha-6'), $3.89(1 \mathrm{H}$, dd, J=1.8, 1.7 and $\left.12.1 \mathrm{~Hz}, \mathrm{H}_{\mathrm{b}}-6^{\prime}\right), 4.93\left(1 \mathrm{H}, \mathrm{d}, \mathrm{J}=7.3 \mathrm{~Hz}, \mathrm{H}-1^{\prime}\right), 5.30\left(1 \mathrm{H}, \mathrm{d}, \mathrm{J}=12.7 \mathrm{~Hz}, \mathrm{H}_{\mathrm{a}}-7\right), 5.42(1 \mathrm{H}, \mathrm{d}$, $\left.\mathrm{J}=12.8 \mathrm{~Hz}, \mathrm{H}_{\mathrm{b}}-7\right), 6.30$ (1H, d, J=15.9 Hz, H-8"), 6.77 (1H, d, J=8.3 Hz, H-5"), 6.94 (1H, dd, J=2.1 and $8.2 \mathrm{~Hz}, \mathrm{H}-6 "), 7.04$ (2H, m. H-4,2"), 7.23 (1H, br d, J=7.8 Hz, H-6), 7.29 $(1 \mathrm{H}, \mathrm{td}, \mathrm{J}=1.6,8.1$ and $7.81 \mathrm{~Hz}, \mathrm{H}-5), 7.36(1 \mathrm{H}, \mathrm{dd}, \mathrm{J}=1.2,0.9$ and $7.4 \mathrm{~Hz}, \mathrm{H}-3), 7.58$ $\left(1 \mathrm{H}, \mathrm{d}, \mathrm{J}=15.9 \mathrm{~Hz}, \mathrm{H}-7^{\prime \prime}\right) .{ }^{13} \mathrm{C}-\mathrm{NMR}\left(100 \mathrm{MHz}, \delta, \mathrm{CD}_{3} \mathrm{OD}\right): 62.55$ (C-6'), 62.62 (C-7), 71.34 (C-4'), 74.99 (C-2'), 78.04 (C-3'), 78.24 (C-5'), 102.92 (C-1'), 115.06 (C-8'), 115.18 (C-2"), 116.52 (C-5"), 116.71 (C-6), 123.08 (C-6"), 123.54 (C-4), 127.22 (C-1"), 127.74 (C-2), 130.41 (C-5), 130.68 (C-3), 146.83 (C-3"), 147.26 (C-7"), 149.66 (C-4"), 157.01 (C-1), 169.31 (C-9").

\section{Antioxidative Activities of the Low Molecular Weight Extractives}

Oxidative stress has been linked with the pathogenesis of many human diseases including cancer, atherosclerosis, and aging (Brawek et al. 2010; Danino et al. 2009; Si et al. 2009a). Currently, there is a growing interest in the research of natural products and plant extracts as antioxidants agents. It has been postulated and verified by researchers that such natural formulations are less toxic and have minimal side effects compared with those chemically synthesized (June et al. 2010; Neo et al. 2010). Therefore, the antioxidative activities of the low molecular weight extractives from Triploid $P$. tomentosa xylem were studied. As oxidative reactions can occur by many mechanisms, with formation of various reactive oxygen radicals, thus in this study, antioxidative activities of Triploid $P$. tomentosa xylem extractives were determined on the basis of two 
classical radical scavenging procedures: DPPH and hydroxyl radical scavenging assays.

\section{$D P P H$ radical scavenging effect}

DPPH scavenging assay is one of the most used methods to study the free radical scavenging ability of antioxidants (Blois 1958). Therefore, we firstly investigate the DPPH radical scavenging effects of the low molecular weight extractives from Triploid $P$. tomentosa xylem. As data summarized in Table 1 , caffeic acid (II) with $\mathrm{IC}_{50}$ value $5.93 \mathrm{uM}$ was clearly more efficient, meanwhile luteolin (IV), salireposide (VI), and populoside (VII) presented IC $_{50}$ values $6.81,6.83$, and $6.80 \mathrm{uM}$, respectively, were very similar to the positive control $\alpha$-tocopherol $\left(\mathrm{IC}_{50} 6.82 \mathrm{uM}\right)$. However, $\rho$-coumaric acid (I), and apigenin (III) gave $\mathrm{IC}_{50}$ values 8.89 and $7.89 \mathrm{uM}$, respectively, were slightly less efficient, and salicortin $\left(\mathbf{V}, \mathrm{IC}_{50}\right.$ value $\left.17.88 \mathrm{uM}\right)$ clearly was not interesting in terms of DPPH radical scavenging.

Table 1. Free Radical Scavenging Effects of the Extractives from Triploid $P$. tomentosa Xylem

\begin{tabular}{lr}
\hline Sample & $I_{50}(\mu \mathrm{M})^{\mathrm{a}}$ \\
\hline I & $8.89 \pm 0.02$ \\
II & $5.93 \pm 0.01$ \\
III & $7.89 \pm 0.02$ \\
IV & $6.81 \pm 0.01$ \\
V & $17.88 \pm 0.01$ \\
VI & $6.83 \pm 0.01$ \\
VII & $6.80 \pm 0.01$ \\
$\alpha$-Tocopherol & $6.82 \pm 0.01$ \\
\hline
\end{tabular}

${ }^{a}$ Values are mean \pm SD of three independent trials.

${ }^{\mathrm{b}}$ Positive control compound.

\section{Hydroxyl radical scavenging effect}

Among all of the reactive oxygen species, the hydroxyl radical plays the most important role in bacterial cell death. Oxidation is one of the key forms of damage (Kohanski et al. 2007). Accordingly, we investigated the antioxidant defense activities of low molecular weight extractives from Triploid $P$. tomentosa xylem on oxidation induced by hydroxyl radicals. From the results demonstrated in Fig. 3, generally speaking, all extractives isolated from Triploid $P$. tomentosa showed dose-dependent antioxidant effects of increasing potential at concentrations from $1 \mathrm{ug} / \mathrm{ml}$ to $10 \mathrm{ug} / \mathrm{ml}$. Caffeic acid (II) at $1 \mu \mathrm{g} / \mathrm{mL}$ and $10 \mu \mathrm{g} / \mathrm{mL}$ showed $77 \%$ and $85 \%$ scavenging ability, respectively, which indicated superior antioxidative ability. Luteolin (IV, 49\% and 58\% at $1 \mathrm{ug} / \mathrm{ml}$ and $10 \mathrm{ug} / \mathrm{ml}$, respectively), salireposide (VI, 54\% and 64\% at $1 \mathrm{ug} / \mathrm{ml}$ and $10 \mathrm{ug} / \mathrm{ml}$, respectively), and populoside (VII, 52\% and $61 \%$ at $1 \mathrm{ug} / \mathrm{ml}$ and $10 \mathrm{ug} / \mathrm{ml}$, respectively) exhibited somewhat equivalent antioxidative potency as $\alpha$-tocopherol (a positive control, $50 \%$ and $60 \%$ at $1 \mathrm{ug} / \mathrm{ml}$ and $10 \mathrm{ug} / \mathrm{ml}$, respectively). By contrast, $\rho$-coumaric acid (I), and apigenin (III) showed weaker effects than $\alpha$-tocopherol, while salicortin (V) exhibited almost no hydroxyl radical scavenging activity. 


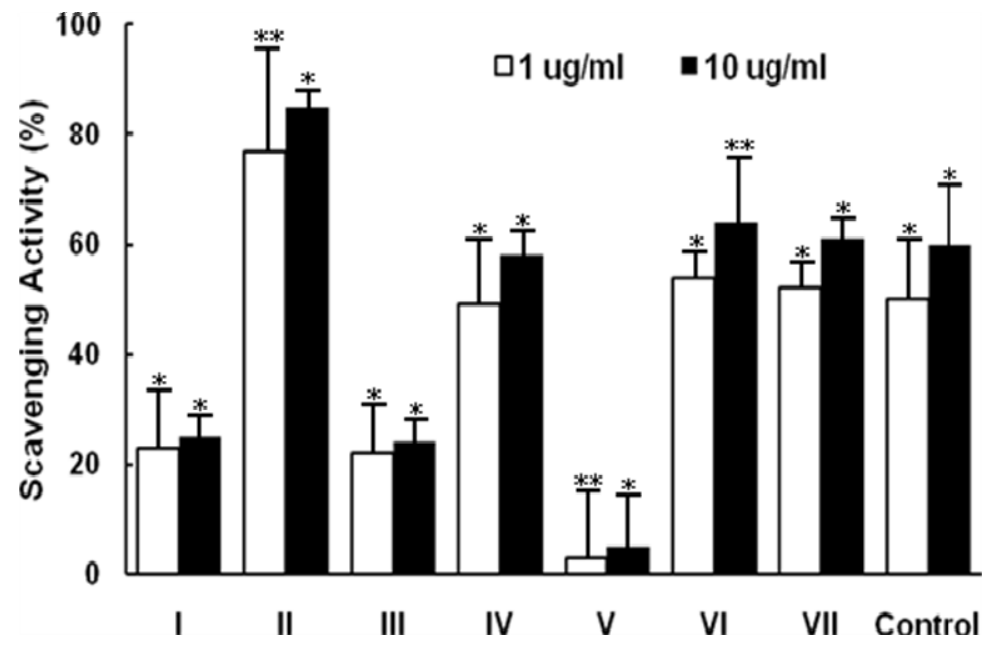

Fig. 3. Hydroxyl radical scavenging activity of the compounds I-VII isolated from Triploid $P$. tomentosa xylem (Values are mean \pm SD of three independent trials; $\alpha$-Tocopherol was used as a positive control)

Relationships between the chemical structure and antioxidative activities of compounds have been determined in many antioxidative activity assays, and they vary with the protocols ( $\mathrm{Si}$ et al. 2008; Wolfe and Liu 2008; Rice-Evans et al. 1996). However, some structure-activity relationships could still be concluded. For example, free hydroxyl groups acts as free radical-scavengers in the flavonoid compounds, and the structures that conferred flavonoids with the most antioxidant activity in the cellular antioxidant activity assays are a 3,4'-O-dihydroxyl group in the B-ring, a 2,3-double bond combined with a 4-keto group in the C-ring, and a 3-hydroxyl group (Wolfe and Liu 2008). For flavones, luteolin (IV) exhibits higher antioxidative activity than apigenin (III) (Wolfe and Liu 2008), which agrees well with our DPPH and hydroxyl radical scavenging assays. The facts above indicated that various low molecular weight extractives in Triploid $P$. tomentosa xylem exhibited significant antioxidative potency and could be an alternative to more toxic synthetic antioxidants presently used in food, pharmaceuticals, and cosmetic products.

\section{CONCLUSIONS}

1. Repeated Chromatographic separation of a $\mathrm{BuOH}$-soluble fraction of Triploid $P$. tomentosa xylem resulted in the isolation of two phenolic acids, $\rho$-coumaric acid (I) and caffeic acid (II), two flavonoids, apigenin (III) and luteolin (IV), and three phenolic glucosides, salicortin (V), salireposide (VI), and populoside (VII). The structures of the isolated phenolic compounds were elucidated and determined on basis of their physiochemical and spectroscopic evidence. This was the first time of reporting the low molecular weight extractives from Triploid P. tomentosa, which will give rise to potential added value development of this fast-growing species. 
2. Antioxidative properties of the extractives of Triploid P. tomentosa xylem were investigated by DPPH and hydroxyl radical scavenging assays. Compounds II exhibited more efficient DPPH and hydroxyl radical scavenging effects than the positive control $\alpha$-tocopherol, and IV, VI, and VII showed the similar activities to the control. Compounds I and III revealed less efficient antioxidative potency when compared with $\alpha$-tocopherol, and $\mathbf{V}$ presented almost no antioxidative capacity in DPPH and hydroxyl radical scavenging assays.

3. Triploid P. tomentosa xylem is a potential new source of natural antioxidants and could be developed for high value-added products.

\section{ACKNOWLEDGMENTS}

The authors express sincere thanks to the National Natural Science Foundation of China (NSFC, No. 31000279), Natural Science Foundation of Tianjin City (09JCYBJC15800) and Foundation for the Development of Science and Technology in Tianjin Universities (No. 20080616) for financial support.

\section{REFERENCES CITED}

Agrawal, P. K. (1989). Carbon-13 NMR of Flavanoids, New York, Elsevier.

Blois, M. S. (1958). "Antioxidant determinations by the use of a stable free radical," Nature 26, 1199-1200.

Brawek, B., Loffler, M., Wagner, K., Huppertz, H. J., Wendling, A. S., Weyerbrock, A., Jackisch, R., and Feuerstein, T. J. (2010). "Reactive oxygen species (ROS) in the human neocortex: Role of aging and cognition," Brain Res. Bull. 81, 484-490.

Casano, G., Dumetre, A., Pannecouque, C., Hutter, S., Azas, N., and Robin, M. (2010). "Anti-HIV and antiplasmodial activity of original flavonoid derivatives," Bioorg. Med. Chem. 18(16), 6012-6023.

Chung, S. K., Osawa, T., and Kawakishi, S. (1997). "Hydroxyl radical-scavenging effects of spices and scavengers from brown mustard (Brassica nigra)," Biosci. Biotech. Biochem. 61(1), 118-123.

Danino,O., Gottlieb, H. E., Grossman, S., and Bergman, M. (2009). “Antioxidant activity of 1,3-dicaffeoylquinic acid isolated from Inula viscosa," Food Res. Int. 42(9), 1273-1280.

Hosoya, T., Yun, Y. S., and Kunugi, A. (2008). "Antioxidant phenylpropanoid glycosides from the leaves of Wasabia japonica," Phytochemistry 69(3), 827-832.

Jung, H. A., Chung, H. Y., Kang, S. S., Hyun, S. K., Kang, H. S., and Choi, J. S. (2010). "A phenolic glucoside isolated from Prunus serrulata var. spontanea and its peroxynitrite scavenging activity," Arch. Pharm. Res. 28(10), 1127-1130.

Kim, J. K., Lee, S. K., Ham, Y. H., and Bae, Y. S. (2002). "Extractives from the barks of Quercus acutissima and Quercus variabilis," J. Korean Forest Energ. 21(1), 41-48. 
Kohanski, M. A., Dwyer, D. J., Hayete, B., Lawrence, C. A., Collins, J. J. (2007). “A common mechanism of cellular death induced by bactericidal antibiotics," Cell 130(5), 797-810.

Kwon, O. G., Kim, S, H., and Chun, B. Y. (1999). "Isolation of antimicrobial components from montan cortex," Korean J. Pharm. 30(3), 340-344.

Lane, N. (2008). “Origins of death," Nature 453(7195), 583-585.

Markham, K. R., and Chari, V. M. (1982). Carbon-13 NMR spectroscopy of flavonoids. In: Harboren J B, Mabry T, Editors. The Flavonoids: Advances in research. London: Chapman and Hall Ltd.

Neo, Y. P., Ariffin, A., Tan, C. P., and Tan, Y. A. (2010). "Phenolic acid analysis and antioxidant activity assessment of oil palm (E. guineensis) fruit extracts," Food Chem. 122(1), 353-359.

Rice-Evans, C. A., Miller, N. J., and Paganga, G. (1996). "Structure antioxidant activity relationships of flavonoids and phenolic-acids," Free Radic. Biol. Med. 20(7), 933-956.

Si, C. L., Kim, K. J., Bae, Y. S., and Li, S. M. (2009a). "Phenolic compounds in the leaves of Populus ussuriensis and their antioxidant activities," Planta Med. 75(10), 1165-1167.

Si, C. L., Deng, X. J., and Liu, Z. (2008). "Structure and activity relationship of antioxidant flavonoids from leaves of Paulownia tomentosa var. tomentosa," 2nd International Papermaking \& Enviroment Conference, Books A and B, 263-266.

Si, C. L., Wu, L., and Zhu, Z. Y. (2009b). "Phenolic glycosides from Populus davidiana bark," Biochem. Syst. Ecol. 37(3), 221-224.

Si, C. L., Xu, J., Kim, J. K., and Bae, Y. S. (2010). “Antioxidant properties and structural analysis of phenolic glucosides from bark of Populus ussuriensis Kom," Wood Sci. Tech. 44(4), in press, DOI: 10.1007/s00226-009-0286-X.

Si, C. L., Xu, Q., Li, S. M., and Liu, Z. (2009c). "Phenolic compounds from Populus davidiana wood," Chem. Nat. Compd. 45(5), 634-636.

Tehila, T. S., Bergman, M., and Grossman, S. (2007). "Cucurbitacin glucosides: Antioxidant and free-radical scavenging activities," Biochem. Biophys. Res. Commun. 364(1), 181-186.

Wolfe, K. L., and Liu, R. H. (2008). "Structure-activity relationships of flavonoids in the cellular antioxidant activity assay," Agric. Food Chem. 56(18), 8404-8411.

Yang, S., Lu, L., and Ni, Y. (2006). "Cloned poplar as a new fibre resource for the Chinese pulp and paper industry," Pulp Paper Canada 107(2), 34-37.

Zhang, A. P., Liu, C. F., and Sun, R. C. (2010). "Fractional isolation and characterization of lignin and hemicelluloses from Triploid of Populus tomentosa Carr.," Ind. Crop. Prod. 31(2), 357-362.

Zhang, X. F., Thuong, P. T., Min, B. S., Ngoc, T. M., Hung, T. M., Lee, I. S., Na, M. K., Seong, Y. H., Song, K. S., and Bae, K. H. (2006). "Phenolic glycosides with antioxidant activity from the stem bark of Populus davidiana," J. Nat. Prod. 69(9), $1370-3137$.

Article submitted: Oct. 11, 2010; Peer review completed: Nov. 24, 2010; Revised version received and accepted: Dec. 2, 2010; Published: Dec. 3, 2010. 